\title{
Monocular and Binocular Visual Function Deficits in Amblyopic Patients with and without Fusion Maldevelopment Nystagmus
}

\author{
Jordan Murray $\mathbb{D}^{1}$ \\ Kiran Garg ${ }^{2}$ \\ Fatema Ghasia ${ }^{3}$ \\ 'Cleveland Clinic Cole Eye Institute, \\ Ophthalmological Research, Cleveland, \\ $\mathrm{OH}$, USA; ${ }^{2}$ Case Western Reserve \\ University School of Medicine, Cleveland, \\ OH, USA; ${ }^{3}$ Cleveland Clinic Cole Eye \\ Institute, Department of Ophthalmology, \\ Cleveland, OH, 44195-000I, USA
}

Purpose: The aim of the study is to examine the association between amblyopia type and the presence of nystagmus on binocular and monocular functions of the fellow (FE) and amblyopic eye (AE).

Methods: We recruited 19 controls and 44 amblyopes (anisometropes $=13$, strabismic $=10$, mixed=21). We measured visual, grating, and vernier acuities and high/low spatial frequency (SF) contrast sensitivities in each eye using a staircase method. Stereoacuity was measured with the Titmus fly test. We recorded fixation eye movements (FEM) using high-resolution video-oculography. Subjects were classified as having either no nystagmus $(n=18)$, fusion maldevelopment nystagmus syndrome (FMNS) $(n=12)$, or nystagmus without any structural anomalies that does not meet the criteria for FMNS or infantile nystagmus $(n=14)$.

Results: Analysis of visual function by clinical amblyopia type showed that patients with strabismus/mixed amblyopia $(\mathrm{F}(2,54)=9.5, \mathrm{p}<0.001)$ were more likely to have poor stereopsis while controlling for AE grating acuity deficit. The FE of patients with anisometropia had greater contrast sensitivity deficits at low $(\mathrm{F}(2,43)=4.4, \mathrm{p}=0.018)$ and high $\mathrm{SF}(\mathrm{F}(2,42)$ $=10.1, \mathrm{p}<0.001)$. Analysis of visual function by FEM characteristics (low SF: $(\mathrm{F}(3,43)=4.3$, $\mathrm{p}=0.010)$ and high SF: $(\mathrm{F}(3,42)=7.1, \mathrm{p}=0.001)$ showed that the FE of patients with FMNS had worse low and high SF contrast sensitivities, whereas those without FMNS had greater contrast sensitivity deficits only at high SF compared to controls. Patients with FMNS (F $(3,54)=12.9, \mathrm{p}<0.001)$ were more likely to have poor stereopsis while controlling for $\mathrm{AE}$ grating acuity deficit compared to patients without FMNS. All amblyopic patients had worse high SF contrast sensitivity of the AE irrespective of type or FEM characteristics (Type = $\mathrm{F}(2,43)=8.8, \mathrm{p}=0.001 ; \mathrm{FEM}$ characteristics $=\mathrm{F}(3,43)=5.1, \mathrm{p}=0.004)$.

Conclusion: The presence of FMNS in patients with strabismic/mixed amblyopia is associated with poor/absent stereopsis. FE deficits vary across amblyopia type. Like FEM abnormalities, visual function deficits are seen in the FE of patients with and without nystagmus.

Keywords: amblyopia, fellow eye, contrast sensitivity, fusion maldevelopment nystagmus, stereopsis

\section{Introduction}

The type of amblyopia affects the constellation of visual deficits seen in the amblyopic eye. Strabismic amblyopes have disproportionately worse visual and vernier acuities and better contrast sensitivity for a given level of grating acuity deficit than anisometropic amblyopes. ${ }^{1,2}$ Psychophysical studies have shown that amblyopia impacts the function of both the amblyopic eye (AE) and fellow eye (FE). ${ }^{2-5}$
Correspondence: Fatema Ghasia Cleveland Clinic Cole Eye Institute, Department of Ophthalmology, 2022, East I05th Street, Cleveland, OH, 44I95-000I, USA

Email fatemaghasia@gmail.com 
Binocular input is combined relatively early in visual processing within the striate cortex (V1) ${ }^{6,7}$ Animal studies have shown that the effects of decorrelated binocular signals on V1 are most significant if they occur at the emergence of stereopsis in early infancy. Early discordant experience results in a more severe breakdown of the excitatory and persistence of inhibitory connections with reduced binocular cells and less precise spatial signaling within the V1 cortex. ${ }^{8}$ This binocular maldevelopment is passed onto downstream extra-striate cortical areas MT/ MST areas responsible for conjugate gaze holding and results in the development of fusion maldevelopment nystagmus (FMNS) ${ }^{8-11}$ Thus, the FE dysfunction could arise from cortical binocular maldevelopment and the resultant FMNS., ${ }^{3,12}$

Increased fixation instability is seen in the $\mathrm{FE}$ and $\mathrm{AE}$ of patients with anisometropic and/or strabismic amblyopia, albeit to a greater degree in the $\mathrm{AE}$, than in controls. ${ }^{13,14}$ We have shown that this instability arises from the presence of nystagmus or due to alterations in physiologic fixation eye movements (FEM), namely fixational saccades and inter-saccadic drifts in patients without nystagmus. ${ }^{13,14}$ Poor stereo-acuity and worse visual acuity deficits are seen in the $\mathrm{AE}$ of patients with greater fixational instability. ${ }^{13-15}$ The relationships between FEM characteristics and amblyopia type on visual functions of the $\mathrm{FE}$ and $\mathrm{AE}$ have not been investigated in the same cohort.

The purpose of our study is to examine the effects of nystagmus and clinical type of amblyopia on binocular and monocular functions of the FE and AE. We hypothesize that amblyopic patients with FMNS will have disproportionately severe binocular function deficits for a given grating acuity deficit. We also hypothesize that, similarly to FEM abnormalities, monocular visual function deficits will be seen in the FE of all amblyopia patients but with greater severity in patients with FMNS.

\section{Materials and Methods}

The Cleveland Clinic institutional review board approved the protocol. Written informed consent was obtained from all participants or their parent/legal guardian per the Declaration of Helsinki. We recruited 63 subjects (19 controls, 44 amblyopes). All subjects were recruited at the Cleveland Clinic. None of the subjects had structural abnormalities of the eye or neurological disorders. The clinical categorization of amblyopia subtype was done per the criteria used in the Pediatric Eye Disease
Investigator Group amblyopia treatment studies. ${ }^{16-18}$ Amblyopia is classified as being associated with strabismus, anisometropia, or both according to the following criteria: 1) Strabismic amblyopia: At least one of the following criteria must be met, and criteria are not met for combined-mechanism amblyopia: a) Heterotropia at a distance and/or near fixation on examination (with or without spectacles)

b) History of strabismus surgery c) documented history of strabismus which is no longer present (and which, in the judgment of the investigator, is the cause of amblyopia) 2) Anisometropic amblyopia: At least one of the following criteria must be met: a) $\geq 0.50 \mathrm{D}$ difference between eyes in spherical equivalent

b) $\geq 1.50 \mathrm{D}$ difference between eyes in astigmatism in any meridian 3) Mixed mechanism amblyopia: Both of the following criteria must be met:

a) criteria for strabismus are met (see above)

b) $\geq 1.00 \mathrm{D}$ difference between eyes in spherical equivalent or $\geq 1.50 \mathrm{D}$ difference between eyes in astigmatism in any meridian. There were 13 anisometropic (AN), 10 strabismic, and 21 mixed amblyopia patients (Supplemental Table S1). The latter two groups were combined for analysis (M/S group). There was no difference in age between controls (mean age $=130.7$ months) and amblyopes (mean age $=158$ months, unpaired $t$-test, $\mathrm{p}=0.15$ ). All the subjects had a comprehensive clinical eye exam with particular emphasis on ocular motility testing and assessing the presence of nystagmus in monocular and binocular viewing conditions. FEM and visual functions were recorded in the lab with best-corrected vision using the subject's own spectacles that were prescribed after the comprehensive clinical eye exam.

\section{Fixation Eye Movements}

A high-resolution infrared imaging-based eye tracker (EyeLink $1000^{\circledR}$, SR Research, Canada) with a spatial resolution of 0.01 degrees at $1000 \mathrm{~Hz}$ was used to record gaze locations with a temporal resolution of $500 \mathrm{~Hz}$. Subjects were instructed to place their head on a chin and forehead rest and fixate on the target while remaining still. An infrared permissive filter was used to block visible light while allowing the non-viewing eye to be tracked. Monocular calibration and validation were performed using a cruciform 5 point constellation. Binocular horizontal and vertical eye positions were measured during $\mathrm{FE}$ and $\mathrm{AE}$ viewing conditions while subjects fixated on a white circular target. The target subtended $0.5^{\circ}$ visual angle on an otherwise black screen $55 \mathrm{~cm}$ 
A NO NYST

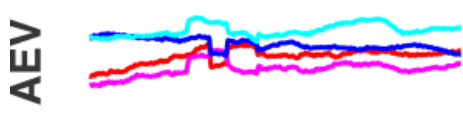

$2^{\circ}$

$1 \mathrm{~s}$

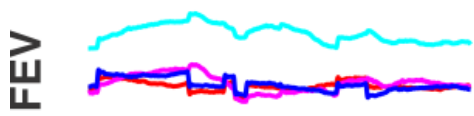

B FMNS
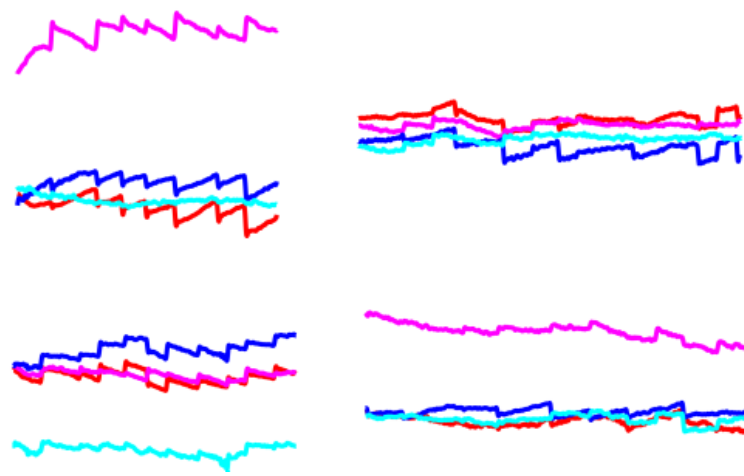

C NO FMNS

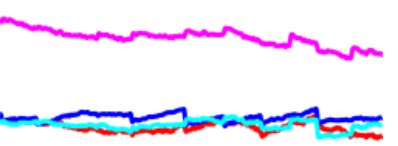

Figure I Gaze positions as a function of time in patients without nystagmus (A), with FMNS (B), and with nystagmus but not FMNS (C) as they fixated on a target monocularly with the amblyopic (top) and fellow (bottom) eyes. Note the large drift amplitudes in the subject without nystagmus. FMNS can be distinguished by the reversal in slow phase direction that occurs when the eye under cover is changed. Red - right horizontal, magenta - right vertical, blue - left horizontal, cyan - left vertical. Rightward and upward movements correspond to the positive vertical axis.

from the subject's eyes for 45 seconds. ${ }^{13,19}$ The eye position data were analyzed using Matlab ${ }^{\mathrm{TM}}$ (MathWorks, Natick, MA). Fixational saccades and quick phases of nystagmus were identified using an unsupervised clustering method. ${ }^{20}$

The FEM traces of the patients were classified based on the presence or absence of nystagmus (Figure 1). ${ }^{14}$ Like control subjects, patients without nystagmus (NN-Figure 1A) exhibited inter-saccadic drifts between fixational saccades. ${ }^{14,21}$ Patients with nystagmus were further divided into those with FMNS (Figure 1B) versus those that did not meet the criteria of FMNS (Figure 1C). The presence of FMNS was determined based on the reversal in nystagmus direction observed under alternate eye monocular viewing such that slow phases of decreasing or constant velocity are nasally directed with respect to the viewing eye. ${ }^{22}$ Patients with nystagmus/nystagmus-like movements who did not exhibit this direction reversal under monocular viewing were characterized as Nystagmus without FMNS (Nyst no FMNS). These patients had jerk nystagmus with dynamic overshoots of quick phases. They differed from Infantile Nystagmus Syndrome patients in that their velocity was decreasing or constant, in contrast to the increasing eye velocity seen in Infantile Nystagmus. Also, patients with nystagmus without FMNS did not have the dissociated vertical deviation frequently seen in FMNS. We classified amblyopes per their FEM characteristics $(\mathrm{NN}=18$, FMNS $=12$, and Nyst no FMNS=14). Table 1 provides the subgroup composition of FEM characteristics per the clinical type of amblyopia.

\section{Visual Functions}

Psykinematix (KyberVision Japan) software was used to generate test stimuli, which were displayed on a monitor with a resolution of $1280 \times 800$ at $60 \mathrm{~Hz}$ with a white luminance of $111 \mathrm{~cd} / \mathrm{m}^{2}$ at a distance of $3.1 \mathrm{~m}$ in a dark room. Amblyopic subjects were recorded under FE and AE viewing while the non-viewing eye was occluded. Control subjects viewed the stimuli binocularly.

1) Contrast Sensitivity: Subjects viewed Gabor patches (spatial sigma $1^{\circ}$ ) at low (4 cycles/deg) and high (14 cycles/ deg) spatial frequencies (SF) and judged the orientation of the patch, which was either horizontal or vertical, selected at random with equal probability. A 3-down-1-up adaptive staircase (proportional step size of $50 \%$ before the first reversal and $25 \%$ increments and $12.5 \%$ decrements thereafter) was used.

2) Visual acuity: Subjects viewed one randomly selected EDTRS optotype with crowding bars- the size was adjusted per a 2-down-1-up staircase with the same step size as above.

Table I Cohort Composition

\begin{tabular}{|l|c|c|c|c|c|}
\hline \multirow{2}{*}{\begin{tabular}{l} 
Amblyopia \\
\cline { 2 - 6 }
\end{tabular}} & \multicolumn{5}{|c|}{ FEM Characteristic } \\
\cline { 2 - 6 } & C & None & $\begin{array}{c}\text { Nyst No } \\
\text { FMNS }\end{array}$ & FMNS & Total \\
\hline AN & 19 & 0 & 0 & 0 & 19 \\
\hline M & 0 & 8 & 5 & 0 & 13 \\
\hline S & 0 & 8 & 7 & 6 & 21 \\
\hline Total & 0 & 2 & 2 & 6 & 10 \\
\hline
\end{tabular}

Note: A breakdown of the cohort by both amblyopia type and FEM characteristic. Abbreviations: C, control; AN, anisometropic; M, mixed; S, strabismic; None, no nystagmus; Nyst No FMNS, nystagmus but not FMNS; FMNS, fusion maldevelopment nystagmus syndrome. 
3) Grating Acuity: Subjects viewed vertical or horizontally oriented Gabor patches (50\% fixed contrast and variable SF adjusted using the same staircase as the contrast experiment) and judged the orientation of the patch. We chose to measure grating acuity at $50 \%$ contrast to obtain a better reference for selecting the spatial frequencies at which to measure contrast sensitivities, which also reduced luminance artifacts from the monitor. ${ }^{23,24}$ We determined the grating acuity threshold of the fellow and amblyopic eye of each participant. We computed the range (upper and lower limit of spatial frequencies) within 1.5 octaves of the $50 \%$ grating acuity threshold. From these ranges, we found that almost all of the study participants could be expected to perform contrast sensitivity staircase procedures at 4 and $14 \mathrm{cpd}$ while viewing with the amblyopic eye. A similar approach has been used previously by Spiegel et al and Yap et $\mathrm{al}^{23,25}$.

4) Vernier acuity: Subjects viewed two vertically oriented bars (dimensions: $1^{\circ} \mathrm{x} 5^{\circ}$ ) displayed with a vertical separation of $0.5^{\circ}$ with the lower bar displayed at a horizontal offset from the centered upper bar with a magnitude determined by a 2-down-1-up staircase and judged the direction of the offset, which they indicated either verbally or by raising a hand.

Each experiment concluded after six reversals, and the thresholds were taken to be the arithmetic mean of the last 4 reversals. Four high SF contrast measurements for subjects with poor visual acuity were discarded due to the inability of the subjects to reliably detect the orientation of the stimuli at $100 \%$ contrast. Contrast thresholds were converted into log values and visual, vernier, and grating acuities to $\log$ MAR values.

Stereopsis: Stereopsis was measured in log arcsecs with the Titmus Fly Test at $40 \mathrm{~cm}$ under binocular viewing. For the purpose of quantitative analysis, subjects with absent stereopsis were assigned a value of 7000 arc seconds.

\section{Statistics}

All analyses were performed in SPSSStatistics (IBM, Armonk, NY), and GraphPad Prism 7 (La Jolla, CA). We used one-way ANOVA to test the significance of amblyopia type and FEM characteristics as factors associated with FE and $\mathrm{AE}$ visual, grating, and vernier acuities. We used twoway repeated-measures ANOVA to assess the differences in acuity measures across amblyopia type and, in a separate analysis, FEM characteristics. For contrast sensitivity and stereopsis, we used ANCOVA to evaluate the significance of amblyopia type and FEM characteristics with AE grating acuity as a covariate for AE functions and FE grating acuity as a covariate for FE functions. Post-hoc analysis was performed with Bonferroni adjustment. The Wilcoxon signedrank test was used to compare the mean grating acuity of incorrectly identified stimuli as a function of the orientation of the stimulus.

\section{Results}

\section{Visual, Grating, and Vernier Acuities}

As expected, AE visual acuity was worse compared to controls across amblyopia type $(\mathrm{F}(2,61)=20.4, \mathrm{p}<0.001)$ and across FEM characteristics ( $F(3,61)=15.3, p<0.001)$ (Table 2). No differences in AE visual acuity were seen between amblyopes across type (AN vs M/S: -0.09 , $95 \mathrm{CI}=[-0.34,0.16], \mathrm{p}=1.0)$ and FEM characteristics $(\mathrm{NN}$ vs Nyst no FMNS:-0.18, 95CI $=[-0.48,0.11], \mathrm{p}=0.6$; Nyst no FMNS vs FMNS: $0.21,95 \mathrm{CI}=[-0.1,0.54], \mathrm{p}=0.4)$.

FE visual acuity varied across type $(\mathrm{F}(2,61)=4.4$, $\mathrm{p}=0.016)$ and trended towards statistical significance across FEM characteristics $(\mathrm{F}(3,61)=2.7, \mathrm{p}=0.05)$ compared to controls. While both anisometropic and mixed/strabismic amblyopes had worse FE visual acuity than controls, the difference between controls (C) and mixed or strabismic amblyopes $(\mathrm{M} / \mathrm{S})$ was significant $(\mathrm{C}$ vs $\mathrm{M} / \mathrm{S}:-.10,95 \mathrm{CI}=$ $[-0.18,0.01], \mathrm{p}=0.018)$. The difference in $\mathrm{C}$ and FMNS group FE visual acuity was just on the cusp of significance (C vs FMNS:-.11, 95CI=[-0.24, 0.00], $\mathrm{p}=0.05$ ).

AE grating acuity was worse compared to controls across type $(\mathrm{F}(2,52)=16.3, \mathrm{p}<0.001)$ and FEM characteristics $(\mathrm{F}$ $(3,51)=12.2, \mathrm{p}<0.001)($ Table 2$)$. However, AE grating acuity deficits were comparable across types (AN vs M/S:-.03, $95 \mathrm{CI}=[-0.23,0.17], \mathrm{p}=1.00)$ and FEM characteristics (NN vs Nyst no FMNS:-.07, 95CI $=[-0.30,0.15], \mathrm{p}=1.00$, NN vs FMNS: $0.09,95 \mathrm{CI}=[-0.16,0.34], \mathrm{p}=1.00$, Nyst no FMNS vs FMNS: $0.16,95 \mathrm{CI}=[-0.10,0.42], \mathrm{p}=0.54)$.

FE grating acuity differed across type $(\mathrm{F}(2,51)=6.7$, $\mathrm{p}<0.01)$ and FEM characteristics $(\mathrm{F}(3,50)=3.1, \mathrm{p}=0.03)$. The FE had worse grating acuity than controls, with significant differences between $\mathrm{C}$ and $\mathrm{M} / \mathrm{S}$ groups ( $\mathrm{C}$ vs $\mathrm{M} /$ $\mathrm{S}:-.13,95 \mathrm{CI}=[-0.21,-.04], \mathrm{p}<0.01)$. For FEM characteristics, the post-hoc comparison between $\mathrm{C}$ and $\mathrm{NN}$ was significant (C vs NN:-.11, 95CI $=[-0.2,0.00], \mathrm{p}=04)$.

The pattern of orientation-dependent defocus depends on the type (hyperopic or myopic) of astigmatism and orientation of astigmatism (ie, with the rule, oblique or against the rule astigmatism). ${ }^{26} \mathrm{We}$ analyzed the mean grating acuity of 
Table 2 Acuity Measurements by Type and Fem Characteristics

\begin{tabular}{|c|c|c|c|}
\hline Clinical Type & Visual Acuity (logMAR) & Grating Acuity (logMAR) & Vernier Acuity (logMAR) \\
\hline \multicolumn{4}{|l|}{ Fellow Eye } \\
\hline Control & $-0.023(0.10)$ & $-0.078(0.06)$ & $-0.086(0.22)$ \\
\hline Anisometropic & $0.007(0.11)$ & $-.031(0.15)$ & $0.038(0.11)$ \\
\hline Mixed/Strabismic & $0.074(0.12)$ & $0.048(0.12)$ & $0.073(0.07)$ \\
\hline \multicolumn{4}{|l|}{ Amblyopic Eye } \\
\hline Control & $-0.023(0.10)$ & $-0.078(0.06)$ & $-0.086(0.22)$ \\
\hline Anisometropic & $0.475(0.31)$ & $0.282(0.24)$ & $0.367(0.30)$ \\
\hline Mixed/Strabismic & $0.566(0.39)$ & $0.313(0.27)$ & $0.472(0.46)$ \\
\hline FEM Characteristics & Visual Acuity (logMAR) & Grating Acuity (logMAR) & Vernier Acuity (logMAR) \\
\hline \multicolumn{4}{|l|}{ Fellow Eye } \\
\hline Control & $-0.023(0.10)$ & $-0.078(0.06)$ & $-0.086(0.22)$ \\
\hline No Nystagmus & $0.052(0.11)$ & $0.037(0.12)$ & $0.122(0.33)$ \\
\hline Nystagmus no FMNS & $0.024(0.13)$ & $0.015(0.17)$ & $-0.054(0.50)$ \\
\hline FMNS & $0.096(0.14)$ & $0.026(0.10)$ & $0.127(0.29)$ \\
\hline \multicolumn{4}{|l|}{ Amblyopic Eye } \\
\hline Control & $-0.023(0.10)$ & $-0.078(0.06)$ & $-0.086(0.22)$ \\
\hline No Nystagmus & $0.486(0.33)$ & $0.299(0.05)$ & $0.377(0.32)$ \\
\hline Nystagmus no FMNS & $0.670(0.4 I)$ & $0.373(0.34)$ & $0.500(0.48)$ \\
\hline FMNS & $0.450(0.35)$ & $0.209(0.17)$ & $0.461(0.47)$ \\
\hline
\end{tabular}

Note: Measurements of acuity (recognition, grating, and vernier) in logMAR by amblyopia type and FEM characteristics.

incorrectly identified stimuli as a function of the orientation of the stimulus. We analyzed the fellow eye and amblyopic eye responses separately per astigmatism type (myopic/ mixed, hyperopic versus emmetropic). We found that a majority of the amblyopic subjects had hyperopic astigmatism, with only 7 patients having myopic astigmatism. The mean grating acuity of incorrectly identified stimuli was comparable across horizontal and vertical orientations during $\mathrm{FE}$ and AE viewing (Table 3). Similar results were seen in controls with emmetropia versus those with myopia. Also, most of the participants had with the rule astigmatism with only 9 subjects with oblique or against the rule astigmatism. Subgroup analysis of amblyopic hyperopic and myopic with the rule astigmats did not reveal any differences between mean grating acuity of incorrectly identified stimuli across horizontal and vertical orientations during FE (Hyperopic with the rule astigmats: horizontal orientation $=0.289 \pm$ 0.159 and vertical orientation $=0.305 \pm 0.137, \mathrm{p}=0.86$ and myopic with the rule astigmats: horizontal orientation $=$ $0.369 \pm 0.062$ and vertical orientation $=0.349 \pm 0.08$, $\mathrm{p}=0.50$ ) and $\mathrm{AE}$ viewing (Hyperopic with the rule astigmats: horizontal orientation $=0.556 \pm 0.255$ and vertical orientation $=0.582 \pm 0.223, \mathrm{p}=0.25$ and myopic with the rule astigmats: horizontal orientation $=0.782 \pm 0.268$ and vertical orientation $=0.767 \pm 0.29, \mathrm{p}>0.99$ ).

The AE had worse vernier acuity thresholds compared to controls across type $(\mathrm{F}(2,58)=13.2, \mathrm{p}<0.001)$ and FEM characteristics $(F(3,57)=8.7, p<0.001)$ (Table 2). However, $\mathrm{AE}$ vernier acuity did not differ significantly between types (AN vs $\mathrm{M} / \mathrm{S}:-.10,95 \mathrm{CI}=[-0.40,0.19], \mathrm{p}=1.00$ ) or across FEM groups (NN vs Nyst no FMNS:-.12, 95CI= $[-0.49,0.24], \mathrm{p}=1.00, \mathrm{NN}$ vs FMNS: $-0.08,95 \mathrm{CI}=[-0.46$, 0.30 ], $\mathrm{p}=1.00$, Nyst no FMNS vs FMNS: 0.03 , $95 \mathrm{CI}=$ $[-0.36,0.44], p=1.0)$. FE vernier acuity did not differ across type $(\mathrm{F}(2,59)=1.2, \mathrm{p}=0.3)$ or $\mathrm{FEM}$ characteristic $(\mathrm{F}(3,58)=1.6, \mathrm{p}=0.17)$ compared to controls.

\section{Comparison of Grating, Vernier, and Visual Acuities Across Type and FEM Characteristics}

To discern the effects of amblyopia type on the visual, vernier, and grating acuities of the $\mathrm{AE}$, we performed two-way 
Table 3 Grating Acuity Miss Values by Astigmatism Type and Orientation

\begin{tabular}{|c|c|c|c|}
\hline Category & Horz Acuity Value & Vert Acuity Value & p value \\
\hline \multicolumn{4}{|l|}{ Controls } \\
\hline Hyperopes & $0.24 I(0.050)$ & $0.288(0.032)$ & - \\
\hline Myopes & $0.234(0.081)$ & $0.240(0.063)$ & $>0.999$ \\
\hline Emmotropes & $0.191(0.034)$ & $0.195(0.079)$ & 0.813 \\
\hline \multicolumn{4}{|c|}{ Amblyopes - Fellow Eye } \\
\hline Hyperopes & $0.289(0.159)$ & $0.296(0.134)$ & 0.877 \\
\hline Myopes & $0.414(0.096)$ & $0.413(0.120)$ & 0.813 \\
\hline Emmotropes & $0.229(0.091)$ & $0.226(0.120)$ & 0.938 \\
\hline \multicolumn{4}{|c|}{ Amblyopes - Amblyopic Eye } \\
\hline Hyperopes & $0.530(0.236)$ & $0.552(0.215)$ & 0.172 \\
\hline Myopes & $0.782(0.268)$ & $0.767(0.296)$ & $>0.999$ \\
\hline Emmotropes & 0.308 & 0.291 & - \\
\hline
\end{tabular}

Notes: Mean logMAR grating acuity values corresponding to missed stimuli of vertical and horizontal orientation for controls and the FE/AE of amblyopes by astigmatism type. These include non-reversal misses and therefore may be larger than the threshold values. $\mathrm{P}$ values are from a Wilcoxon matched-pairs signed rank test. The null hypothesis is that the mean acuity values of the horizontal and vertical stimuli are equivalent.

repeated-measures ANOVA. We found that the main effect of AE acuity is statistically significant, sphericity assumed ( $F$ $(2100)=10.6, p<0.001)$ however, the interaction between acuity of the AE and amblyopia type narrowly missed significance, sphericity assumed $(\mathrm{F}(4100)=2.3, \mathrm{p}=0.062)$. Posthoc comparisons identified differences between visual and grating $(0.165,95 \mathrm{CI}=[0.08,0.25], \mathrm{p}<0.001)$ and between visual and vernier $(0.09,95 \mathrm{CI}=[0.01,0.18], \mathrm{p}=0.021)$ with no differences between grating and vernier acuities $(-0.07$, $95 \mathrm{CI}=[-0.17,0.03], \mathrm{p}=0.25)$. We did a similar analysis to determine the effects of amblyopia type on the acuity of the FE and found no significance, sphericity assumed (F (2100) $=0.72, \mathrm{p}=0.49$ ) and no interaction between acuity of the FE and amblyopia type, sphericity assumed $(F(4100)=0.208$, $\mathrm{p}=0.93)$.

A two-way repeated-measures ANOVA was performed to determine the effects of FEM characteristics on visual, vernier, and grating acuities of the AE. We found that the main effect of the acuity of the AE is significant, sphericity assumed ( $F(2,98)$ $=14.7, \mathrm{p}<0.001$ ) however, the interaction between acuity of the $\mathrm{AE}$ and FEM characteristics fell short of significance, sphericity assumed $(\mathrm{F}(6,98)=2.0, \mathrm{p}=0.070)$. Post-hoc comparisons identified differences between visual and grating $(0.19$, $95 \mathrm{CI}=[0.11,0.27], \mathrm{p}<0.001)$, grating and vernier $(-0.11$, $95 \mathrm{CI}=[-0.20,-0.01], \mathrm{p}=0.026)$ and visual and vernier acuities $(0.08,95 \mathrm{CI}=[0.00,0.16], \mathrm{p}=0.046)$. We did a similar analysis to determine the effects of FEM characteristics on the acuity of the FE and found no significance for the acuity of the FE, sphericity assumed $(\mathrm{F} \quad(2,98)=0.57, \mathrm{p}=0.567)$, and no interaction between acuity of the FE and FEM characteristics, sphericity assumed $(\mathrm{F}(6,98)=1.43, \mathrm{p}=0.211)$.

\section{Contrast Sensitivities}

Figure 2 plots the AE contrast sensitivity at high and low SF as a function of type (Figure 2A) and FEM characteristics (Figure 2B). We found that the AE contrast sensitivity at high SF differed significantly across types $(\mathrm{F}(2,43)=8.8$, $\mathrm{p}=0.001)$ with AE grating acuity as a covariate. The covariate also had a strong effect $(\mathrm{F}(1,43)=55.9, \mathrm{p}<0.001)$ on contrast sensitivity. Pairwise differences between the controls and the other groups were significant (C vs AN:-.58, $95 \mathrm{CI}=[-0.94,-.23], \mathrm{p}<0.001, \mathrm{C}$ vs $\mathrm{M} / \mathrm{S}:-.42,95 \mathrm{CI}=[-0.72$,$.11], \mathrm{p}=0.004)$. Amblyopia type was associated with differences in contrast sensitivity at low $\operatorname{SF}(F(2,50)=5.7$, $\mathrm{p}=0.006)$, as was the covariate $(\mathrm{F}(1,50)=70.0, \mathrm{p}<0.001)$. The AN threshold was significantly higher than both $\mathrm{C}$ and $\mathrm{M} / \mathrm{S}$ groups (C vs AN:-.33, 95CI $=[-0.64,-.01], \mathrm{p}=0.037, \mathrm{AN}$ vs $\mathrm{M} / \mathrm{S}: 0.32,95 \mathrm{CI}=[0.07,0.57], \mathrm{p}<0.001)$. While controlling for grating acuity, the FEM characteristic $(\mathrm{F}(3,43)=5.1$, $\mathrm{p}=0.004$ ) significantly affected AE contrast sensitivity at high SF. The covariate also had a strong effect $(\mathrm{F}(1,43)$ $=56.3, \mathrm{p}<0.001)$. Differences between controls and the no nystagmus and nystagmus without FMNS groups were significant (C vs NN:-.41, 95CI $=[-0.81,-.014], \mathrm{p}=0.039, \mathrm{C}$ vs No FMNS:-.53, 95CI=[-0.91,-.15], $\mathrm{p}=0.002)$, while the difference between controls and FMNS patients trended towards significance $(\mathrm{C}$ vs FMNS:-.40, 95CI $=[-0.80$, 0.003 ], $\mathrm{p}=0.05)$. AE contrast sensitivity at low SF did not 
A AE Contrast By Type

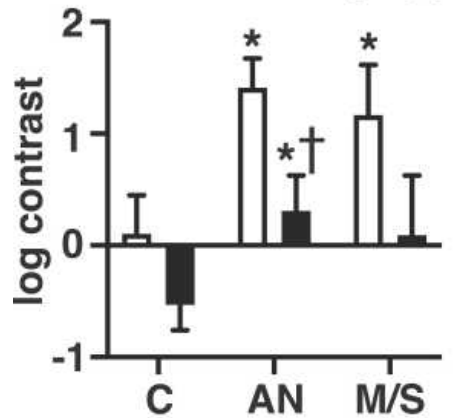

B AE Contrast By FEM

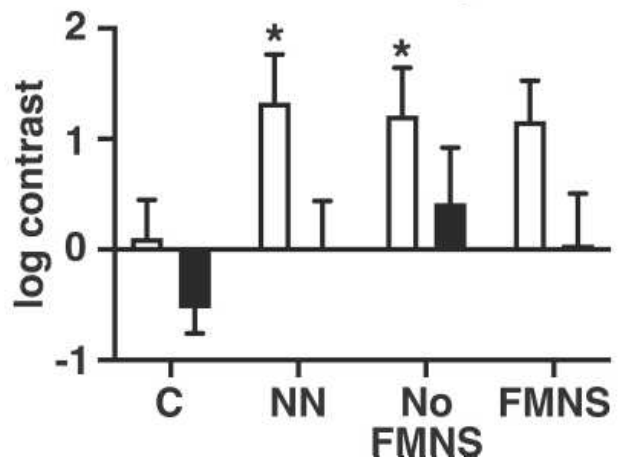

Figure 2 Mean and standard error of the mean for amblyopic eye log percentage Michelson contrast thresholds measured at 4 (black bars) and 14 (white bars) cycles per degree for subjects grouped by clinical type $(\mathbf{A})$ and FEM characteristic $(\mathbf{B})$. Single asterisks and daggers denote significant $(p<0.05)$ differences relative to the control and mixed/strabismic groups, respectively, after controlling for AE grating acuity and applying Bonferroni correction.

differ significantly between FEM characteristic groups after controlling AE grating acuity $(\mathrm{F}(3,51)=2.0, \mathrm{p}=0.12)$.

Figure 3 plots the FE contrast sensitivity at high and low $\mathrm{SF}$ as a function of type (Figure $3 \mathrm{~A}$ ) and FEM characteristics (Figure 3B). FE contrast sensitivity at high SF varied significantly across amblyopia types with FE grating acuity as a covariate $(\mathrm{F}(2,42)=10.1, \mathrm{p}<0.001)$. The covariate was also significant $(\mathrm{F}(1,42)=7.6, \mathrm{p}=0.008)$. Post-hoc comparisons between controls and both amblyopia groups were significant $(\mathrm{C}$ vs $\mathrm{AN}:-.54,95 \mathrm{CI}=[-0.87,-.20], \mathrm{p}=0.001$, $\mathrm{C}$ vs $\mathrm{M} / \mathrm{S}:-.45,95 \mathrm{CI}=[-0.75,-.14], \mathrm{p}=0.002)$. Amblyopia type was a significant factor in FE contrast sensitivity at low SF $(\mathrm{F}(2,43)=4.4, \mathrm{p}=0.018)$. The pairwise difference was significant between controls and anisometropes ( $\mathrm{C}$ vs AN:-.32, 95CI $=[-0.60,-.04], \mathrm{p}=0.021)$. FEM characteristic was associated with significant differences in FE contrast sensitivity at high $\mathrm{SF}(\mathrm{F}(3,42)=7.1, \mathrm{p}=0.001)$. The covariate, FE grating acuity, also had a significant effect $(\mathrm{F}(1,42)$
$=5.9, \mathrm{p}=0.019)$. Pairwise differences between the control group and all amblyopic FEM groups were significant ( $\mathrm{C}$ vs $\mathrm{NN}:-.53,95 \mathrm{CI}=[-0.91,-0.16], \mathrm{p}=0.002, \mathrm{C}$ vs NoFMNS: $-0.39, \quad 95 \mathrm{CI}=[-0.76,-.02], \mathrm{p}=0.03, \mathrm{C}$ vs FMNS:-.57, $95 \mathrm{CI}=[-0.99,-.15], \mathrm{p}=0.003)$. Contrast sensitivity at low SF was associated with FEM characteristic $(F(3,43)=4.3$, $\mathrm{p}=0.010$ ). The difference between controls and the FMNS group was significant (C vs FMNS:-.37, 95CI $=[-0.68,-.06]$, $\mathrm{p}=0.012$ ).

With AE grating acuity as a covariate, amblyopia type had a significant effect on stereopsis deficits ( $F(2,54)=9.5$, $\mathrm{p}<0.001$ )(Figure 4). Stereoacuities were significantly worse in mixed/strabismic amblyopes compared with both anisometropic amblyopes and controls ( $\mathrm{C}$ vs $\mathrm{M} / \mathrm{S}:-1.2,95 \mathrm{CI}=$ $[-1.9,-.48], \mathrm{p}<0.001, \mathrm{AN}$ vs $\mathrm{M} / \mathrm{S}:-.7,95 \mathrm{CI}=[-1.3,-.04]$, $\mathrm{p}=0.034)$. FEM characteristic had a significant effect on stereopsis $(\mathrm{F}(3,54)=12.9, \mathrm{p}<0.001)$ with AE grating acuity as a covariate. Pairwise differences were significant between

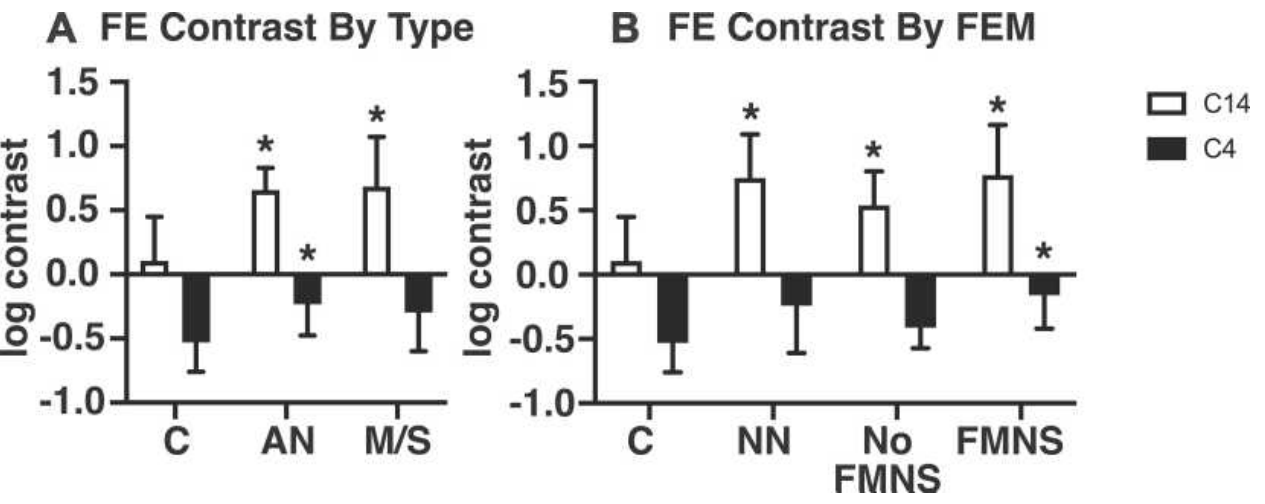

Figure 3 Mean and standard error of the mean for fellow eye log percentage Michelson contrast thresholds measured at 4 (black bars) and 14 (white bars) cycles per degree for subjects grouped by clinical type $(\mathbf{A})$ and FEM characteristics $(\mathbf{B})$. Single asterisks denote significant $(p<0.05)$ differences relative to the control group after controlling for FE grating acuity and applying Bonferroni correction. 


\section{A Stereopsis By Type}

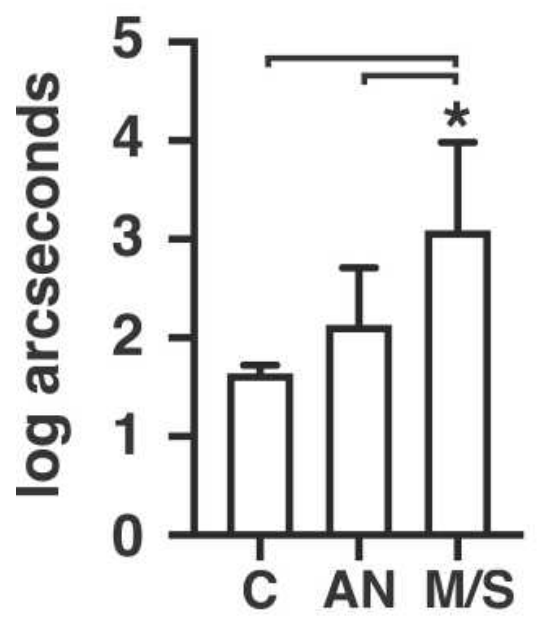

B Stereopsis By FEM

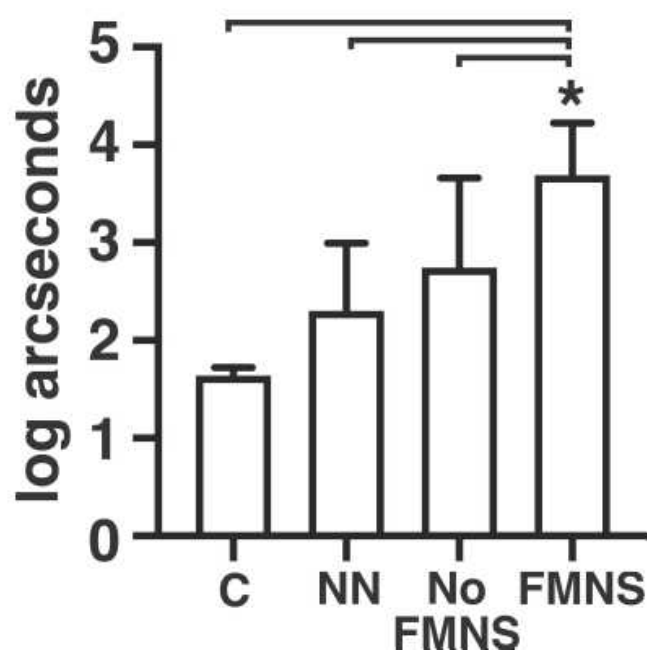

Figure 4 Mean and standard error of the mean for stereopsis in log arcseconds grouped by type (A) and FEM characteristic (B). Single asterisks denote statistically significant $(\mathrm{P}<0.05)$ differences compared to the groups indicated by the brackets after controlling for AE grating acuity and applying Bonferroni correction.

amblyopes with FMNS and all other groups (FMNS vs C: $1.7,95 \mathrm{CI}=[.93,2.6], \mathrm{p}<0.001$, FMNS vs $\mathrm{NN}: 1.3,95 \mathrm{CI}=$ $[.58,2.1], \mathrm{p}<0.001$, FMNS vs No FMN: 0.88, 95CI=[0.075, 1.6], $\mathrm{p}=0.025)$ and between controls and amblyopes without nystagmus (C vs NN:-.88, 95CI=[-1.7,-.03], p=0.037). We found that 11/12 FMNS patients had absent stereopsis. Thus, we did a subgroup analysis of the effects of the presence of FMNS on stereopsis deficits in patients with strabismic/ mixed amblyopia and found that FEM characteristic had a significant effect $(F(2,24)=5.7, p<0.01)$. Strabismic/ mixed amblyopia patients without nystagmus had better stereoacuity with a pairwise comparison showing significant differences between no nystagmus and FMNS group (NN vs FMNS:-1.25, 95CI $=[-2.20,-.30], \mathrm{p}<0.01)$ after controlling for grating acuity deficit. We performed subgroup analysis across FEM characteristics within the M/S group and the AN group for contrast and stereopsis, but only the stereopsis result across FEM presented above was significant, perhaps owing to the small size of the subgroups and resultant low power.

\section{Discussion}

We found that the pattern of visual function deficits in the $\mathrm{FE}$ and $\mathrm{AE}$ and binocular functions differ based on both clinical type and FEM characteristics. Patients with microstrabismus and FMNS were more likely to have absent stereopsis despite having only mild to moderate amblyopia than strabismic patients without FMNS. On the other hand, anisometropic and strabismic/mixed amblyopes without nystagmus and those with nystagmus without FMNS had stereopsis deficits predicted by the severity of the AE grating acuity deficit. The FE of patients with FMNS had worse optotype, low and high SF contrast sensitivities, whereas those without FMNS had greater contrast sensitivity deficits at high SF than controls. All amblyopic patients had worse high SF contrast sensitivity of the AE that cannot be attributed to the FEM characteristics or grating acuity deficit of the AE.

Several studies have evaluated visual function deficits per the clinical type of amblyopia in both adults ${ }^{1}$ and children. ${ }^{2,15,27,28}$ In agreement with previous studies, we found that strabismic/mixed patients were more likely to have poor stereopsis with less pronounced contrast sensitivity deficits with thresholds affected only at high SF when accounting for the AE's grating acuity deficits. The reverse was seen in the $\mathrm{AE}$ of anisometropic amblyopes with greater contrast sensitivity deficits at both low and high SF and stereopsis deficits predicted by the AE's grating acuity deficit. The high SF contrast measurements are a sensitive marker of the central visual system dysfunction. ${ }^{29,30}$ Thus, the reduced thresholds at high SF across all groups reflect cortically mediated vision loss in amblyopia. Similar to prior studies, we found that the grating acuity deficits were less pronounced in the $\mathrm{AE}$ than vernier and visual acuity deficits. ${ }^{1,2}$ However, we did not find differences in the ratios of 
visual/grating acuity deficits across type of amblyopia. This could be attributed to our study population of patients with varying amblyopia severity and the lower contrast of $50 \%$ used to test the grating acuity thresholds. ${ }^{28}$ Previous studies have reported a horizontal effect in non-amblyopic children and an oblique effect in non-amblyopic adults. ${ }^{31-34}$ In our study, we measured grating acuities at horizontal and vertical orientations and found no systematic bias in thresholds per the orientation of the stimulus in either controls or amblyopic subjects. Offering a binary choice between two orientations enabled us to reduce testing time and simplified the task for our pediatric participants. However, the lack of testing at oblique orientations could have limited the ability to detect horizontal and oblique effects and resulted in the absence of meridional anisotropy in our study participants. We also found less impairment of visual functions in the FE of the anisometropic than the mixed/strabismic group. We found that the contrast sensitivity thresholds at high SF were nine-fold better in controls than FE of patients with mixed/strabismic amblyopia. We also found that the grating acuity thresholds of FE of strabismic/mixed amblyopes were lower by an average of $32 \%$, and the visual acuity was three-fold worse compared to controls. Besides high SF contrast impairment, the contrast sensitivity thresholds at low SF were two-fold better in controls than anisometropes. Binocular summation has been reported in controls with better thresholds under binocular than monocular viewing. The binocular summation for grating acuity is reported to be $5 \%, 7 \%$ for visual acuity at $100 \%$ contrast and the contrast sensitivity thresholds have a ratio of 1.5 for control binocular/preferred eye and 1.6 for control binocular/ non-preferred eye. ${ }^{35-37}$ Thus, the reported deficits in the FE are greater than can be accounted for by binocular summation alone in controls.

FEM abnormalities have been reported in the $\mathrm{FE}$ and $\mathrm{AE}$ with differing features between patients with and without strabismus. ${ }^{13,14,19,38}$ It is known that monocular visual acuity can worsen in FMNS patients due to an increase in nystagmus intensity. ${ }^{39,40}$ Thus, FE dysfunction is often attributed to the presence of nystagmus. None of the studies to date have evaluated contrast, grating, vernier, visual acuities of $\mathrm{FE}$ and $\mathrm{AE}$, stereopsis deficits in conjunction with FEM abnormalities in the same cohort. A few studies have evaluated the correlation between the severity of visual and stereo-acuity deficits as a function of AE fixation instability as measured by bivariate contour ellipse area. ${ }^{13,41,42}$ These studies have excluded patients with nystagmus or have not separately analyzed patients with and without nystagmus. This is particularly important, as we know that the analysis of eye position traces is necessary to identify FMNS. ${ }^{14,15,43}$ In the current study, we found that the FE of amblyopic patients with and without nystagmus exhibits visual function abnormalities, especially in FMNS patients. FE grating acuity thresholds of patients without nystagmus were lower by an average of $58 \%$. The mean visual acuity of FMNS patients was four-fold worse than controls. The contrast sensitivity thresholds at low and high SF were three-fold and nine-fold better, respectively in controls than FMNS patients. We have previously reported FEM abnormalities in patients without nystagmus, such as increased eye position variance during inter-saccadic drifts in the FE. ${ }^{13}$ It is possible that the increased drifts could arise from diminished visual feedback and the resultant loss of inputs that normally optimize the performance of the neural network for gaze holding. ${ }^{44}$

\section{Conclusions}

In agreement with prior studies, patients with microstrabismus and amblyopia who had FMNS were more likely to have poor or absent stereopsis than those without FMNS ${ }^{8,22}$ Fellow eye visual function deficits vary across amblyopia type and are seen in the fellow eye of patients with and without nystagmus. Our study emphasizes the importance of FEM analysis and highlights the relationship between visual afferent and efferent system deficits seen in amblyopia. Future studies of larger cohorts evaluating FEM abnormalities and visual functions within each clinical subtype are warranted to assess the impact of amblyopia.

\section{Abbreviations}

FEM, fixational eye movements; FMNS, fusion maldevelopment nystagmus syndrome; NN, no nystagmus used in reference to an amblyopia patient without nystagmus; AE, amblyopic eye; FE, fellow eye; SF, spatial frequency.

\section{Data Sharing Statement}

The data upon which the analyses submitted for publication are based will be made available upon request. The data consist of gaze positions measured during gaze holding and the results of visual function measurements. 


\section{Ethics Approval and Informed Consent}

The Cleveland Clinic institutional review board (Federalwide Assurance: FWA 00005367) approved the protocol (12-915). Written informed consent was obtained from all participants or their parent/legal guardian per the Declaration of Helsinki.

\section{Consent for Publication}

All materials submitted for publication can be published. The authors have seen the article contents and consent to its publication. Signed consent forms from all authors can be furnished upon request.

\section{Acknowledgments}

The authors would like to thank the National Eye Institute, Research to Prevent Blindness, the Blind Children's Center, the CWRU school of Medicine, and the Cleveland Clinic for their financial support.

\section{Author Contributions}

All authors made a significant contribution to the work reported, whether that is in the conception, study design, execution, acquisition of data, analysis and interpretation, or in all these areas; took part in drafting, revising or critically reviewing the article; gave final approval of the version to be published; have agreed on the journal to which the article has been submitted; and agree to be accountable for all aspects of the work.

\section{Funding}

NEI T32: 5 T32 EY 24236-4 (JM). CWRU School of Medicine, Dean's Summer Research Award (KG). Research to Prevent Blindness, Walt Disney Amblyopia Award (FG). Research to Prevent Blindness, Unrestricted Block Grant CCLCM (FG). CWRT CTSC Pilot Grant Program (FG). Cleveland Clinic RPC Grant (FG).

\section{Disclosure}

None of the authors have any conflicts of interest to report.

\section{References}

1. Levi DM, Klein S. Differences in vernier discrimination for gratings between strabismic and anisometropic amblyopes. Invest Ophthalmol Vis Sci. 1982;23(3):398-407.

2. McKee SP, Levi DM, Movshon JA. The pattern of visual deficits in amblyopia. J Vis. 2003;3(5):380-405. doi:10.1167/3.5.5
3. Birch EE, Kelly KR, Giaschi DE. Fellow eye deficits in amblyopia. $J$ Binocular Vision Ocular Motility. 2019;69(3):116-125. doi:10.1080/2576117X.2019.1624440

4. Chen D, Otero-Millan J, Kumar P, Shaikh AG, Ghasia FF. Visual search in amblyopia: abnormal fixational eye movements and suboptimal sampling strategies. Invest Ophthalmol Vis Sci. 2018;59 (11):4506-4517. doi:10.1167/iovs.18-24794

5. Kelly KR, Cheng-Patel CS, Jost RM, Wang YZ, Birch EE. Fixation instability during binocular viewing in anisometropic and strabismic children. Exp Eye Res. 2018;183:29-37. doi:10.1016/j. exer.2018.07.013

6. Hubel D, Wiesel T. Ferrier lecture - functional architecture of the macaque monkey visual cortex. Proc $R$ Soc Lond B Biol Sci. 1977;198(1130):1-59. doi:10.1098/rspb.1977.0085

7. Tychsen L, Burkhalter A. Neuroanatomic abnormalities of primary visual cortex in macaque monkeys with infantile esotropia: preliminary results. J Pediatr Ophthalmol Strabismus. 1995;32(5):323-328.

8. Tychsen L, Richards M, Wong A, Foeller P, Bradley D, Burkhalter A. The neural mechanism for latent (fusion maldevelopment) nystagmus. J Neuroophthalmol. 2010;30:276-283. doi:10.1097/ WNO.0b013e3181dfa9ca

9. Calcutt C, Murray ADN. Untreated essential infantile esotropia: factors affecting the development of amblyopia. Eye. 1998;30 (3):276-283.

10. Gresty M, Metcalfe T, Timms C, Elston J, Lee J, Liu C. Neurology of latent nystagmus. Brain. 1992;115(5):1303-1321. doi:10.1093/brain/ 115.5.1303

11. Bedell H, Flom M. Bilateral oculomotor abnormalities in strabismic amblyopes: evidence for a common central mechanism. Doc Ophthalmol. 1985;59:309-321. doi:10.1007/BF00159166

12. Meier K, Giaschi D. Unilateral amblyopia affects two eyes: fellow eye deficits in amblyopia. Invest Ophthalmol Vis Sci. 2017;58 (3):1779-1800. doi:10.1167/iovs.16-20964

13. Shaikh AG, Otero-Millan J, Kumar P, Ghasia FF. Abnormal fixational eye movements in amblyopia. PLoS One. 2016;11(3): e0149953. doi:10.1371/journal.pone.0149953

14. Kang S, Beylergil S, Otero-Millan J, Shaikh A, Ghasia F. Fixational eye movement waveforms in amblyopia: characteristics of fast and slow eye movements. JEMR. 2019;12(6). doi:10.16910/jemr.12.6.9

15. Birch EE. Amblyopia and binocular vision. Prog Retin Eye Res. 2013;33:67-84. doi:10.1016/j.preteyeres.2012.11.001

16. Manh VM, Holmes JM, Lazar EL, et al. A randomized trial of a binocular iPad game versus part-time patching in children aged 13 to 16 years with amblyopia. Am J Ophthalmol. 2018;186:104-115. doi:10.1016/j.ajo.2017.11.017

17. Pediatric Eye Disease Investigator Group. A randomized trial of near versus distance activities while patching for amblyopia in children aged 3 to less than 7 years. Ophthalmology. 2008;115 (11):2071-2078. doi:10.1016/j.ophtha.2008.06.031

18. Cotter SA, Weakley JDR, Strauber SF, et al. Pharmacological plus optical penalization treatment for amblyopia. Arch Ophthalmol. 2009;127(1):22-30.

19. Ghasia FF, Otero-Millan J, Shaikh AG. Abnormal fixational eye movements in strabismus. Br J Ophthalmol. 2018;102(2):253-259. doi:10.1136/bjophthalmol-2017-310346

20. Otero-Millan J, Castro JL, Macknik SL, Martinez-Conde S. Unsupervised clustering method to detect microsaccades. $J$ Vis. 2014;14(2). doi:10.1167/14.2.18

21. Shaikh AG, Ghasia FF. Fixational saccades are more disconjugate in adults than in children. PLoS One. 2017;12(4):e0175295. doi:10.1371/journal.pone. 0175295

22. Abadi R, Scallan C. Waveform characteristics of manifest latent nystagmus. Invest Ophthalmol Vis Sci. 2000;41(12):3805-3817.

23. Yap TP, Luu CD, Suttle C, Chia A, Boon MY. Effect of stimulus orientation on visual function in children with refractive amblyopia. Invest Ophthalmol Vis Sci. 2020;61(5):5. doi:10.1167/iovs.61.5.5 
24. Brigell M, Bach M, Barber C, Moskowitz A, Robson J; Calibration Standard Committee of the International Society for Clinical Electrophysiology of V. Guidelines for calibration of stimulus and recording parameters used in clinical electrophysiology of vision. Doc Ophthalmol. 2003;107:185-193. doi:10.1023/A:1026244901657

25. Spiegel DP, Byblow WD, Hess RF, Thompson B. Anodal transcranial direct current stimulation transiently improves contrast sensitivity and normalizes visual cortex activation in individuals with amblyopia. Neurorehabil Neural Repair. 2013;27(8):760-769. doi:10.1177/1545968313491006

26. Harvey EM, Dobson V, Miller JM, Clifford-Donaldson CE. Amblyopia in astigmatic children: patterns of deficits. Vision Res. 2007;47(3):315-326. doi:10.1016/j.visres.2006.11.008

27. Zhao W, Jia W, Chen G, et al. A complete investigation of monocular and binocular functions in clinically treated amblyopia. Sci Rep. 2017;7.

28. Birch EE, Swanson WH. Hyperacuity deficits in anisometropic and strabismic amblyopes with known ages of onset. Vision Res. 2000;40 (9):1035-1040. doi:10.1016/S0042-6989(00)00011-0

29. Davison H. Electrophysiology of the retina: response to stimulation and the transduction process. In: Davison H, editor. Physiology of the Eye. 5 ed. London: Macmillan Press; 1990:279-304.

30. Bishop P. Processing of visual information within the retinostriate system. In: Bishop P, editor. The Handbook of Physiology: The Nervous System III (Section 1). Baltimore: Waverly Press; 1984:341-424.

31. Yap TP, Luu CD, Suttle CM, Chia A, Boon MY. Electrophysiological and psychophysical studies of meridional anisotropies in children with and without astigmatism. Invest Ophthalmol Vis Sci. 2019;60 (6):1906-1913. doi:10.1167/iovs.18-25924

32. Brown AM, Lindsey DT, Cammenga JG, Giannone PJ, Stenger MR. The contrast sensitivity of the newborn human infant. Invest Ophthalmol Vis Sci. 2015;56(1):625-632. doi:10.1167/iovs.14-14757

33. Murray IJ, Elliott SL, Pallikaris A, Werner JS, Choi S, Tahir HJ. The oblique effect has an optical component: orientation-specific contrast thresholds after correction of high-order aberrations. J Vis. 2010;10 (11):10. doi: $10.1167 / 10.11 .10$
34. Furmanski CS, Engel SA. An oblique effect in human primary visual cortex. Nat Neurosci. 2000;3(6):535-536. doi:10.1038/75702

35. Zlatkova M, Anderson R, Ennis F. Binocular summation for grating detection and resolution in foveal and peripheral vision. Vision Res. 2001;41(24):3093-3100. doi:10.1016/S0042-6989(01)00191-2

36. Campbell F, Green D. Monocular versus binocular visual acuity. Nature. 1965;208(5006):191-192. doi:10.1038/208191a0

37. Home R. Binocular summation: a study of contrast sensitivity, visual acuity and recognition. Vision Res. 1978;18(5):579-585. doi:10.1016/ 0042-6989(78)90206-7

38. Ciuffreda K, Kenyon R, Stark L. Saccadic intrusions in strabismus. Arch Ophthalmol. 1979;97(9):1673-1679. doi:10.1001/ archopht.1979.01020020241012

39. Dorman K. Binocular versus monocular acuity in a patient with latent nystagmus. J Am Optom Assoc. 1982;53(6):485-486.

40. Moore D. Visual acuity assessment in latent nystagmus. In: LenkSchafer M, editor. Orthoptic Horizons. Transactions of the Sixth International Orthoptic Congress. Harrogate, UK; 1987:487-491.

41. Chung ST, Kumar G, Li RW, Levi DM. Characteristics of fixational eye movements in amblyopia: limitations on fixation stability and acuity? Vision Res. 2015;114:87-99. doi:10.1016/j. visres.2015.01.016

42. Gonzalez EG, Wong AM, Niechwiej-Szwedo E, Tarita-Nistor L, Steinbach MJ. Eye position stability in amblyopia and in normal binocular vision. Invest Ophthalmol Vis Sci. 2012;53(9):5386-5394. doi:10.1167/iovs. 12-9941

43. Scaramuzzi M, Murray J, Otero-Millan J, Nucci P, Shaikh A, Ghasia F. Part time patching treatment outcomes in children with amblyopia with and without fusion maldevelopment nystagmus: an eye movement study. PLoS One. 2020;15(8). doi:10.1371/journal. pone. 0237346

44. Schneider R, Thurtell M, Eisele S, Lincoff N, Bala E, Leight R. Neurological basis for eye movements of the blind. PLoS One. $2013 ; 8$.
Eye and Brain

\section{Publish your work in this journal}

Eye and Brain is an international, peer-reviewed, open access journal focusing on clinical and experimental research in the field of neuroophthalmology. All aspects of patient care are addressed within the journal as well as basic research. Papers covering original research, basic science, clinical and epidemiological studies, reviews and

Submit your manuscript here: https://www.dovepress.com/eye-and-brain-journal evaluations, guidelines, expert opinion and commentary, case reports and extended reports are welcome. The manuscript management system is completely online and includes a very quick and fair peerreview system, which is all easy to use. Visit http://www.dovepress. com/testimonials.php to read real quotes from published authors. 